DE92 009478

\title{
PERFORMANCE OF THE FRONT END ELECTRONICS AND DATA ACQUISITION SYSTEM FOR THE SLD CHERENKOV RING IMAGING DETECTOF*
}

\author{
K. Abe, ${ }^{a}$ P. Antilogus, ${ }^{b,[1]}$ D. Aston, ${ }^{b}$ K. Baird, ${ }^{c}$ A. Bean, ${ }^{d}$ T. Bienz, ${ }^{b,[2]}$ F. Bird, ${ }^{b},[3]$ D. O. Caldwell, ${ }^{d}$ \\ M. Cavalli-Sforza, ${ }^{e}$ P. Coyle, ${ }^{e,[4]}$ D. Coyne, ${ }^{e}$ S. Dasu, ${ }^{b}$ S. Dolinsky, ${ }^{b,[7]}$ A. d'Oliveira, ${ }^{f,[8]} \mathrm{J}$. Duboscq, ${ }^{d}$ \\ W. Dunwoodie, ${ }^{b}$ P. Gagnon, ${ }^{e}$ G. Hallewell, ${ }^{b}$ K. Hasegawa, ${ }^{a}$ J. Hoeflich, J. Huber, ${ }^{d}$ P. Jacques ${ }^{c}$ R. A. Johnson, \\ M. Kalelkar, ${ }^{c}$ H. Kawahara, ${ }^{b}$ Y. Kwon, ${ }^{b}$ D.W.G.S. Leith, ${ }^{b}$ X. Liu, ${ }^{e}$ A. Lu, ${ }^{d}$ D. Marshall, ${ }^{b}$ J. Martinez, ${ }^{b}$ L. Mathys, ${ }^{d}$ \\ S. McHugh, ${ }^{d}$ B. Meadows, ${ }^{f}$ D. Muller, ${ }^{b}$ T. Nagamine ${ }^{b}$ M. Nussbaum, ${ }^{f}$ G. Oxoby, ${ }^{b}$ T. J. Pavel ${ }^{b}$ R. Plano, ${ }^{c}$ \\ B. Ratcliff, P. Rensing, A. K. S. Santha, ${ }^{f}$ D. Schultz, J. Shank, ${ }^{b}$ S. Shapiro, ${ }^{b}$ A. Shoup, ${ }^{f,[b]}$ C. Simopoulos, ${ }^{b}$ \\ M.D. Sokoloff, ${ }^{f}$ E. Solodov, ${ }^{b,[7]}$ P. Stamer, ${ }^{c}$ P. Stiles, ${ }^{b}$ I. Stockdale, ${ }^{f},[6]$ F. Suekane, ${ }^{a}$ N. Toge, ${ }^{b}$ J. Va'vra, ${ }^{b}$ \\ J. S. Whitaker, D. A. Williams, ${ }^{e}$ S. H. Williams, ${ }^{b}$ M. Witherell, ${ }^{d}$ R. J. Wilson, ${ }^{d}$ G. Word, ${ }^{c}$ S. Yellin, ${ }^{d}$ H. Yuta ${ }^{a}$

\begin{abstract}
${ }^{a}$ Department of Physics, Tohoku University, Aramaki, Sendai 980, JAPAN
${ }^{b}$ Stanford Linear Accelerator Center, Stanford, CA 94309, USA

${ }^{\circ}$ Serin Physics Laboratory, Rutgers University, P.O. Box 849, Piscataway, NJ 08855, USA

Department of Physics, University of California, Santa Barbara, CA 93106, USA

¿Santa Cruz Inst. for Particle Physics, University of California, Santa Cruz, CA 95064, USA

1 Department of Physics, University of Cincinnati, Cincinnati, OH 45221, USA

${ }^{9}$ Department of Physics, Boston University, Boston, MA 02215, USA
\end{abstract}

Abstract

The front end electronics and data acquisition system for the SLD barrel Cherenkov Ring Imaging Detector (CRID) are described. This electronics must provide a $1 \%$ charge division measurement with a maximum acceptable noise level of 2000 electrons (rms). Noise and system performance results are presented for the initial SLD engineering run data.

\section{INTRODUCTION}

The SLD is an $e^{+} e^{-}$spectrometer designed for $Z^{0}$ physics. It consists of five major detector subsystems for charged particle tracking and momentum measurement, particle identification and total energy measurement [1,2]. The Cherenkov Ring Imaging Detector (CRID) provides particle identification. Cherenkov photons generated by the passage of charged particles through liquid and gaseous radiators enter drift boxes with quartz windows and ionize a drift gas mixture. The resulting photo-electrons drift in a uniform electric field and are detected on $10 \mathrm{~cm}$ long $7 \mu \mathrm{m}$ diameter carbon sense wires [3]. Two coordinates of the point of origin of the photoelectron are determined by the wire address and the drift time. Charge division, achieved by means of an amplifier on each end of the $40 \mathrm{k} \Omega$ wire, allows the calculation of the third coordinate which is along the wire.

ॠWork supported in part by Department of Energy contract DE-AC03-76SF00515, and by National Science Foundation grants PHY 88-13669 and PHY 88-13018.

Present Addresses:

1 Inst. de Physique Nucleaire, $43 \mathrm{Bd}$. du 11 novembre 1918 , 69622 Villeurbanne, France.

2 Dept. of Physics \& Astronomy, University of Iowa, lowa City, IA 52242 .

${ }^{13}$ SSC Laboratory, 2550 Beckleymeade Avenue, Suite 125, Dallas, TX 75237 .

${ }^{4}$ Centre de Physique des Particules, Faculte des Sciences de Luminy, 13288 Marseille, France.

5 Dept. of Physics, University of California, Irvine, CA 92717.

6 ASA Ames Research Center, Mail Stop 258-6, Moffett Field, CA $94035-1000$.

Permanent Addresses:

7 Inst. of Nuclear Physics, Novosibirsk 90, 630090, USSR.

8 Universidade Estadual Paulista, UNESP

Campus De Guaratingueta, 12500 Sao Paulo-SP Brazil.
The specifications for this system require a $1 \%$ charge division measurement on single $e^{-}$pulses from a wire chamber in the presence of much larger signals. Fast recovery $(\sim 1 \mu \mathrm{s})$ from saturating pulses and noise levels below 2000 electrons (rms) for single electron pulses are required. The construction of the front end electronics and data acquisition system is complete and the components are being installed [4]. This paper provides a description of the front end electronics and data acquisition system along with initial results from bench tests, cosmic ray tests and the SLD engineering run [5].

\section{DESCRIPTION OF THE ELECTRONICS AND DATA ACQUISITION SYSTEM}

There are 40 detectors in the barrel CRID, each having 93 sense wires on a $3.2 \mathrm{~mm}$ pitch. Every wire is instrumented on each end (inner and outer) with a single channel hybrid preamplifier [6], hybrid analog memory unit (HAMU) [7], 12 bit analog to digital converter (ADC), and calibration circuitry for channel-to-channel gain and pedestal variations. A FASTBUS plant controls the system and processes the data from the CRID detectors. When requested, the digitized data is sent via fiberoptics to waveform sampling modules (WSMs) where the data is demultiplexed, compacted (snipped) and sent to a deconvolution (pulse finding) routine to find pulses and calculate the three coordinates. Figure 1 shows a block diagram of the overall acquisition system for one CRID sense wire. To eliminate a cable plant carrying fast, low-level analog signals, the front end electronics (from preamps through digitization) are mounted directly onto the detector. Figure 2 is a photograph of a front end electronics stack, demonstrating the nhysical constraints and density of the electronics necessary in this design.

The preamplifier functions are implemented using a low-noise JFET, followed by an analog semicustom integrated circuit that includes the remaining bipolar circuitry. Channel calibration is done to an accuracy of $0.5 \%$. For this, a circuit is provided on each preamplifier to generate an analog reference step. Active trimming is needed, (a) to bring the low gain and high gain DC offsets of the hybrid output to the $1.2 \mathrm{~V} \mathrm{DC}$ necessary for the signal to be within 


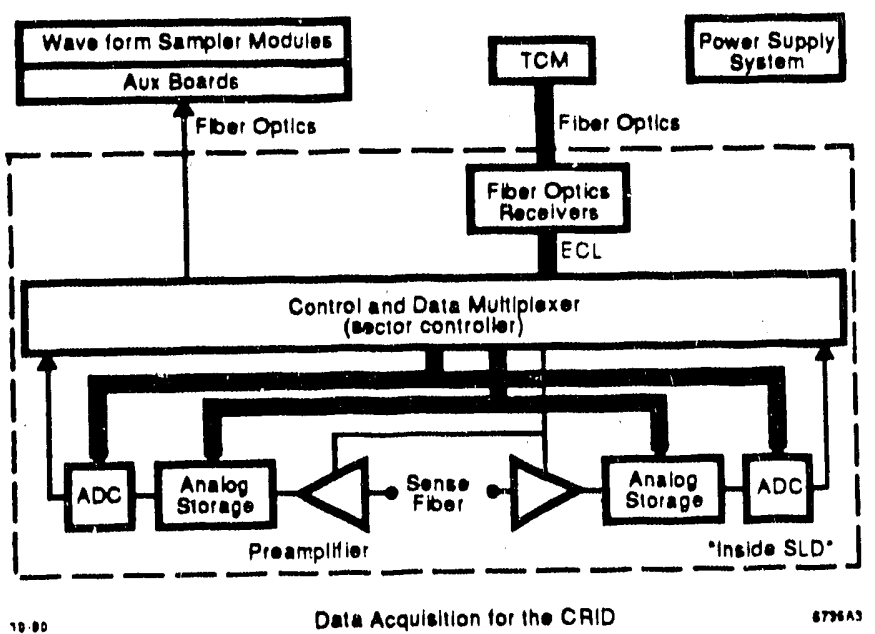

Fig. 1. Block diagram of the data acquisition system for one CRID sense wire.

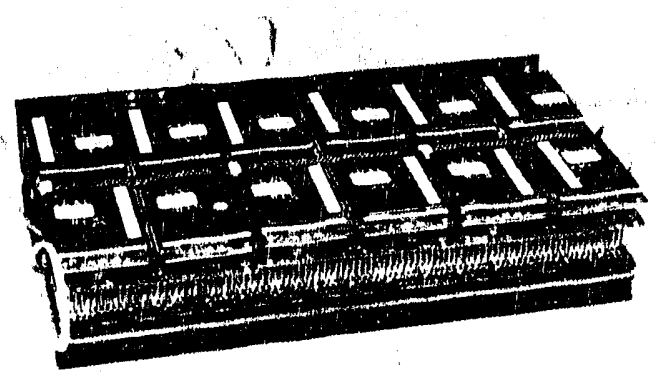

Fig. 2. Photograph of the front-end electronics which demonstrates the severe mechanical constraints.

the dynamic range of the HAMU, and (b) to adjust the calibration circuitry. Each channel has two AMUs, each providing 256 time samples (buckets) of 67 nanoseconds for a total of 512 time samples. A DC calibration is performed on the HAMU to compensate for bucket-to-bucket pedestal variations and nonlinear effects. The ADC boards provide the analog-to-digital conversion for the HAMU output, and parallel to serial conversion of the digitized data. A sector controller provides the interface between two detectors' electronics and the general SLD data acquisition system.

\section{PERFORMANCE}

Before mounting each of the 40 "stacks" of electronics on the CRID detector, they are required to meet certain criteria on the bench. The analog calibration circuitry is checked for functionality by having the correct pulse height observed on every channel. After the HAMU DC calibration, the DC level is checked for range and linearity as read out by the 12 bit ADC. An average noise per channel on the pedestal is also calculated by finding the standard deviation over 1000 pulses on each of the time buckets and then averaging the 512 resulting numbers. This noise is checked to be less than approximately 10 ADC counts or

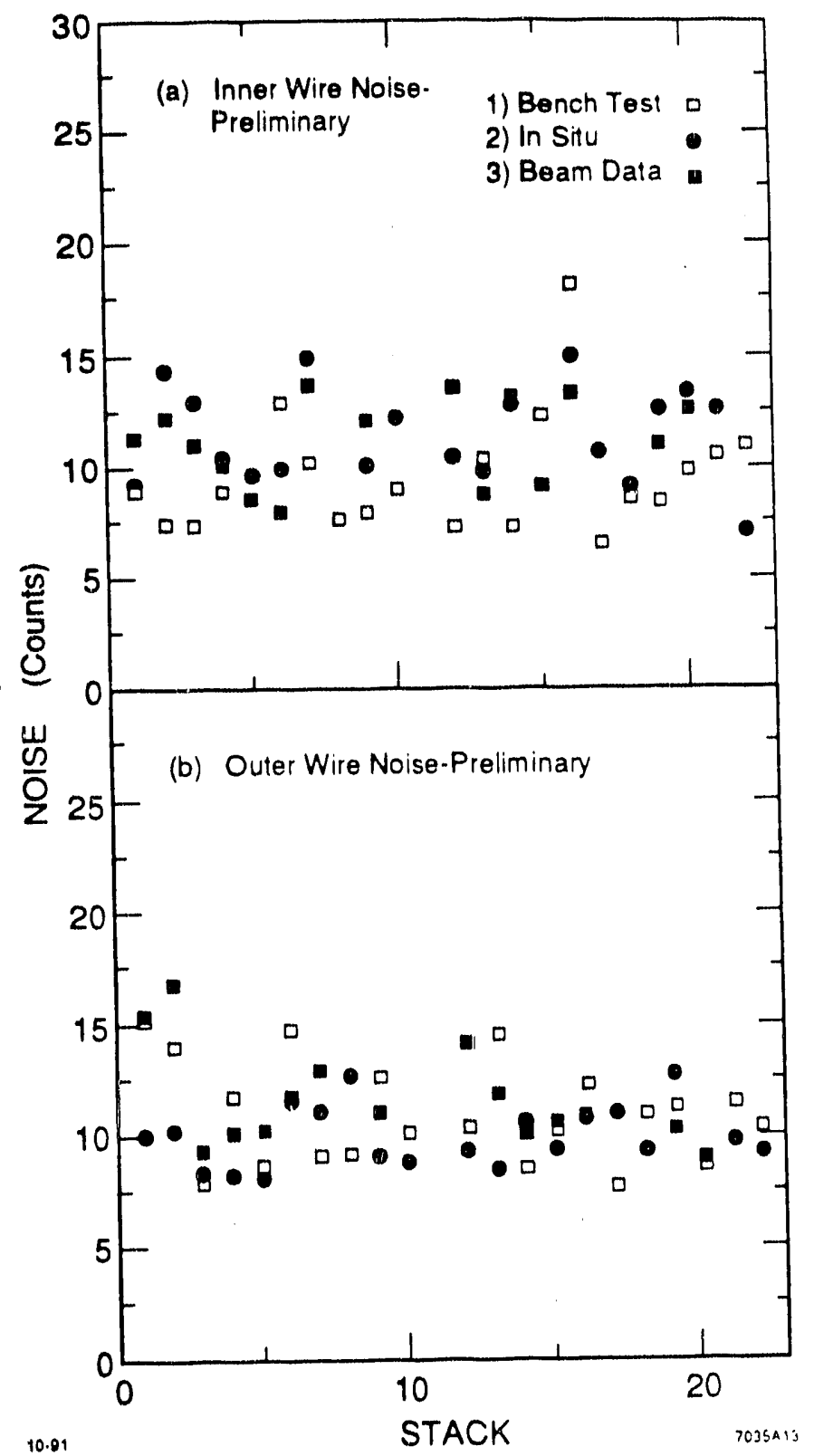

Fig. 3. Preliminary noise measurements on: (a) innet and. (b) outer ends of a wire for, 1) bench tests, 2) in. silu huchet noise and 3) pedestal noise of colliding beam data

$2000 e^{-}$. The resulis for both ends of one wire on each of the 22 packages installed during the SLD enginetrint run are shown in Figure 3 . There is a point-to-point sytematic error of $\pm 2 A D C$ counts associated with all noin measurements which is not shown.

After the packages were installed in SLD, noise is determined using online and offline algorithms. Both meth-. ods use 6 pedestal buckets prior to an injected calibration pulse. In the online algorithm, the standard deviation for each bucket is found and then the six measurements are averaged. The second data set is taken from diagnostic triggers depicted in Figure 3. In the offline algorithm. a mean pedestal is calculated on each event and the devidtion of each bucket from this mean is taken as the noisc: In Figure 3, data set 3 shows the results of this methad 
for real $Z^{0}$ events. All measurements agree within the \pm 2 count systematic error, showing that there is no significant increase in the noise when the electronics is installed in SLD, even when the full detector is running and taking $Z^{0}$ data. The design goal of noise low enough for $1 \%$ charge division resolution has been met, and may be exceeded, as a recent change in the feedback circuit to the $\mathrm{H} A \mathrm{MU}$ has been implemented which reduces the average bench test noise from ten to eight counts.

Uninteresting data are cut out using the WSMs. The WSMs contain a digital correction unit (DCU) which applies a threshold cut on each end of each wire and only then passes this "snipped" data to the deconvolution stage. The threshold cut of 140 counts above the nominal pedestal value is applied with eight preceding and 20 following buckets on both ends snipped. With this cut, an average event with 22 of the 40 detectors in use, 10 kilobytes are transmitted to the online computer. In principle, deconvolution can be performed in the WSMs as well, but for the SLD engineering run, the deconvolution computation was performed on the online VAX computer. With the previously mentioned improvements in our noise, the improvements to the pedestal stability as a function of trigger rate, and the movement of the deconvolution computation to the WSM, we expect to decrease the threshold cut considerably and simultaneously reduce the amount of data transmitted to the VAX for the full complement of electronics expected for the next run.

The CRID preamplifiers are designed so that the pulse from a CRID detector closely approximates the shape $1 e^{-t / \tau}$. This shape permits the deconvolution of the amplifier response from the sampled data with a simple linear relation using precalculated coefficients. To find the pulses, the algorithm performs a simple amplitude threshold search on the deconvolved data. Once found, a hit is classified by how many samples arc over threshold. Figure $4 \mathrm{a}$ shows the averaged output frorn a single preamplifier for identified single electron pulses in colliding beam dat a taken during the engineering run. One can see that the fit is excellent by examining the residuals shown in Figure 4b. Figure 5 shows the distribution of the pulse shap constant $\tau$ for all hit wires. The measured value for $\tau$ found is consistent with the preamplifier design.

Cosmic ray data has been taken to demonstrate that a minimum ionizing particle (MIP) signature can be recognized by the electronics. Figure 6 a shows a typical MIP raw data output with a large signal amplitude. Examples of wire profiles taken during colliding beam events are shown in Figure $6 \mathrm{~b}-\mathrm{d}$. One can see well behaved single electron pulses in Figure $6 \mathrm{~b}$. Figures $6 \mathrm{c}$ and $6 \mathrm{~d}$ show the effect of nearest-neighbor cross talk from a MIP signal which results in a negative going amplitude. All single electron pulses shown are found by ihe deconvolution algorithm. However, the negative going, cross talk signal sometimes causes the algorithm to find spurious pulses. The algorithm is robust against large (multielectron) pulses, as indicated in Figures $7 \mathrm{a}$ and $7 \mathrm{~b}$, which present raw profile data. The large pulses result from a slow (5.6 MeV) electron spiralling down a drift box in the SLD magnetic field. Such complicated waveforms are difficult to analyze with threshold electronics. The pulses have approximately \& overlapping electrons each, and the track is nicely reconstructed as seen in Figure $7 \mathrm{c}$.

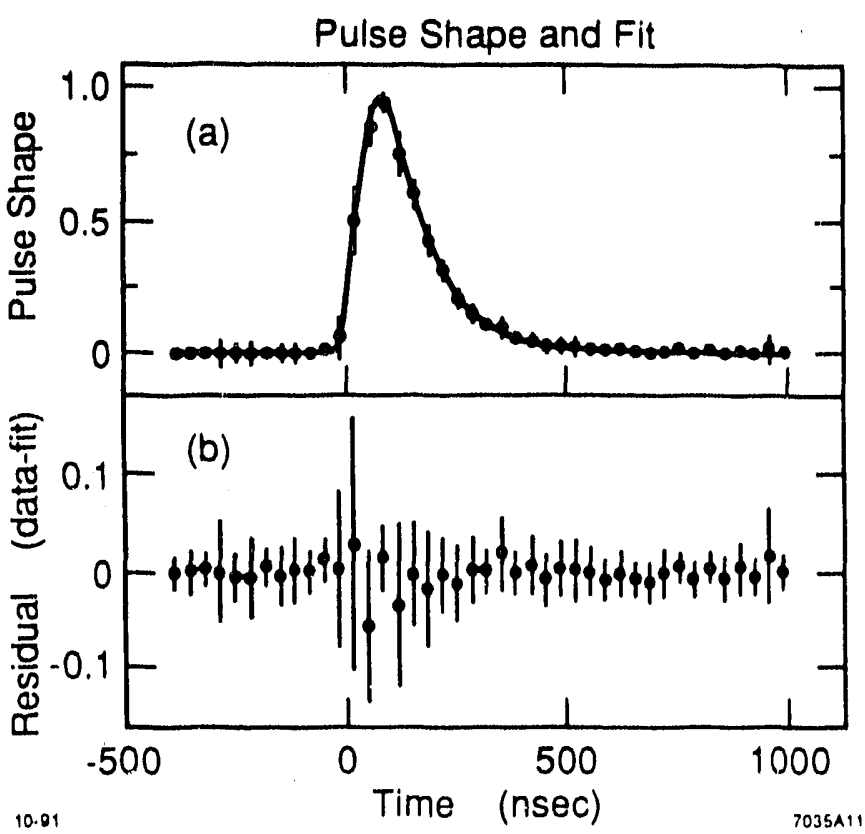

Fig. 4. (a) Pulse shape of single preamplifier from colliding beam events and, (b) residuals of fit performed using deconvolution algorithm demonstrating the good fit to our biodel.

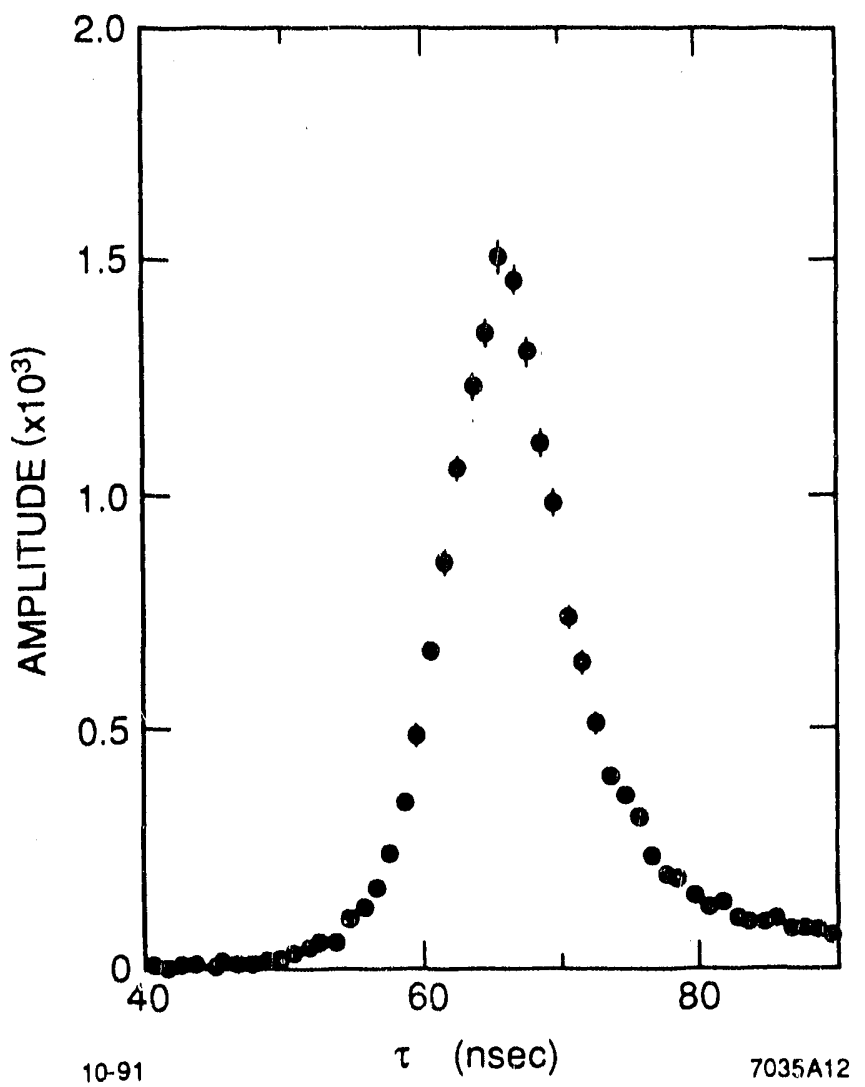

Fig. 5. Distribution of the pulse shape constant $r$ for all hit wires with a mean consistent with the design values

\section{CONCLUSIONS}

We have successfully developed and constructed an electronic data acquisition system for the SLD CRID 


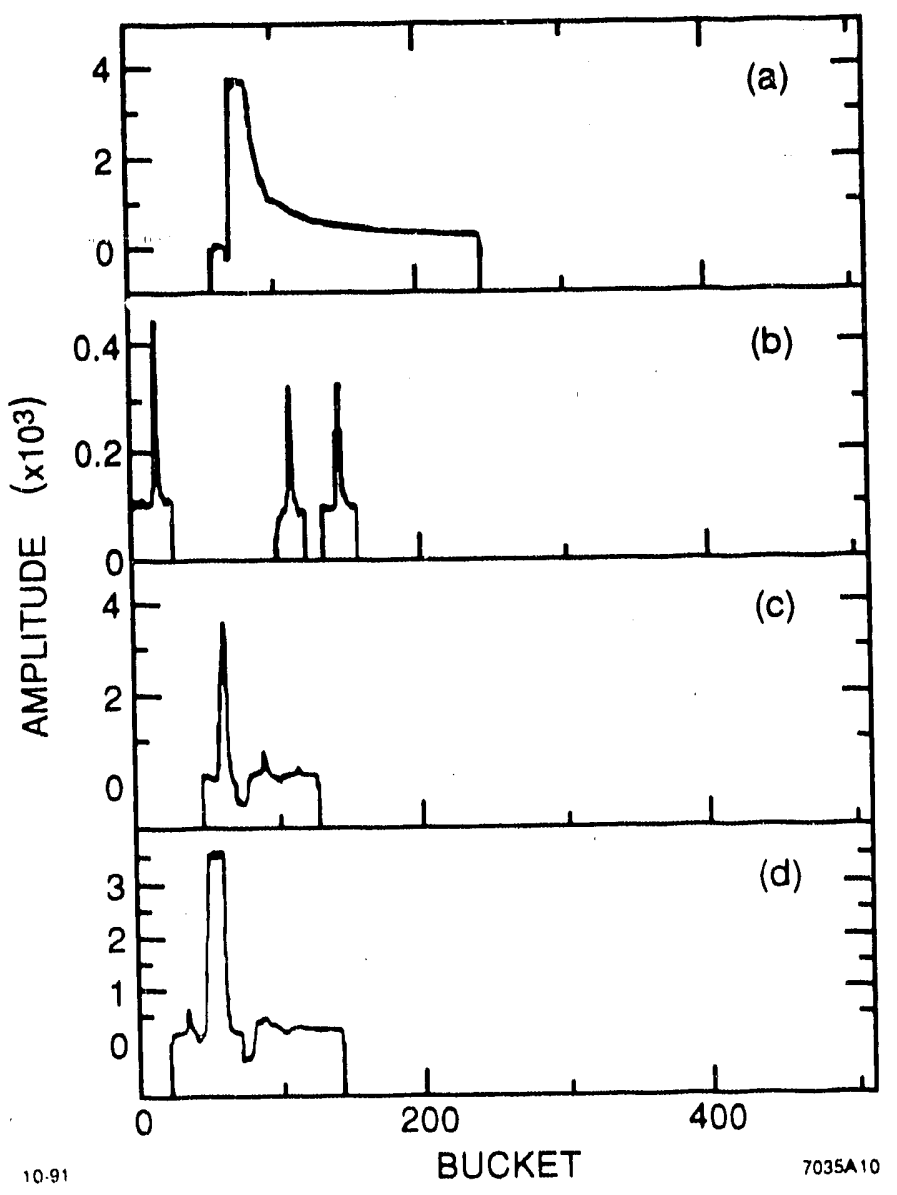

Fig. 6. Example wire readout for, (a) large $d E / d x$ or minimum ionizing particle pulse which show the recovery over a few hundred nanoseconds to the pedestal value, (b) well beliaved single electron pulses, and (c) and (d) events with MIIP signals and nearest neighbor cross talk

Bencli tests and beam data demonstrate that we have reached our design goal for noise performance. Further improvement is expected. The data acquisition system and pulse deconvolution technique perform well and with a high efficiency.

\section{R.EFERENCES}

(1) SLD Design Report SLAC-273, UC-34D, May 1984, and revisions; D. Aston et al., Nucl. Instrum. Methods, A283, 58:2-589 (1989), SLAC-PUB-4795.

[2] M. Breidenbach et al., "A Status Report on the SLD Data Acquisition System," IEEE Trans. Nucl. Sci. NS-36, 23 (1989).

[3] J. Va'Vra et al., "Construction and Initial Operation of a Proportional Wire Detector for Use in a Cherenkov Ring Imaging System, ${ }^{n}$ IEEE Trans. Nucl. Sci. NS-35, 487 (1988).
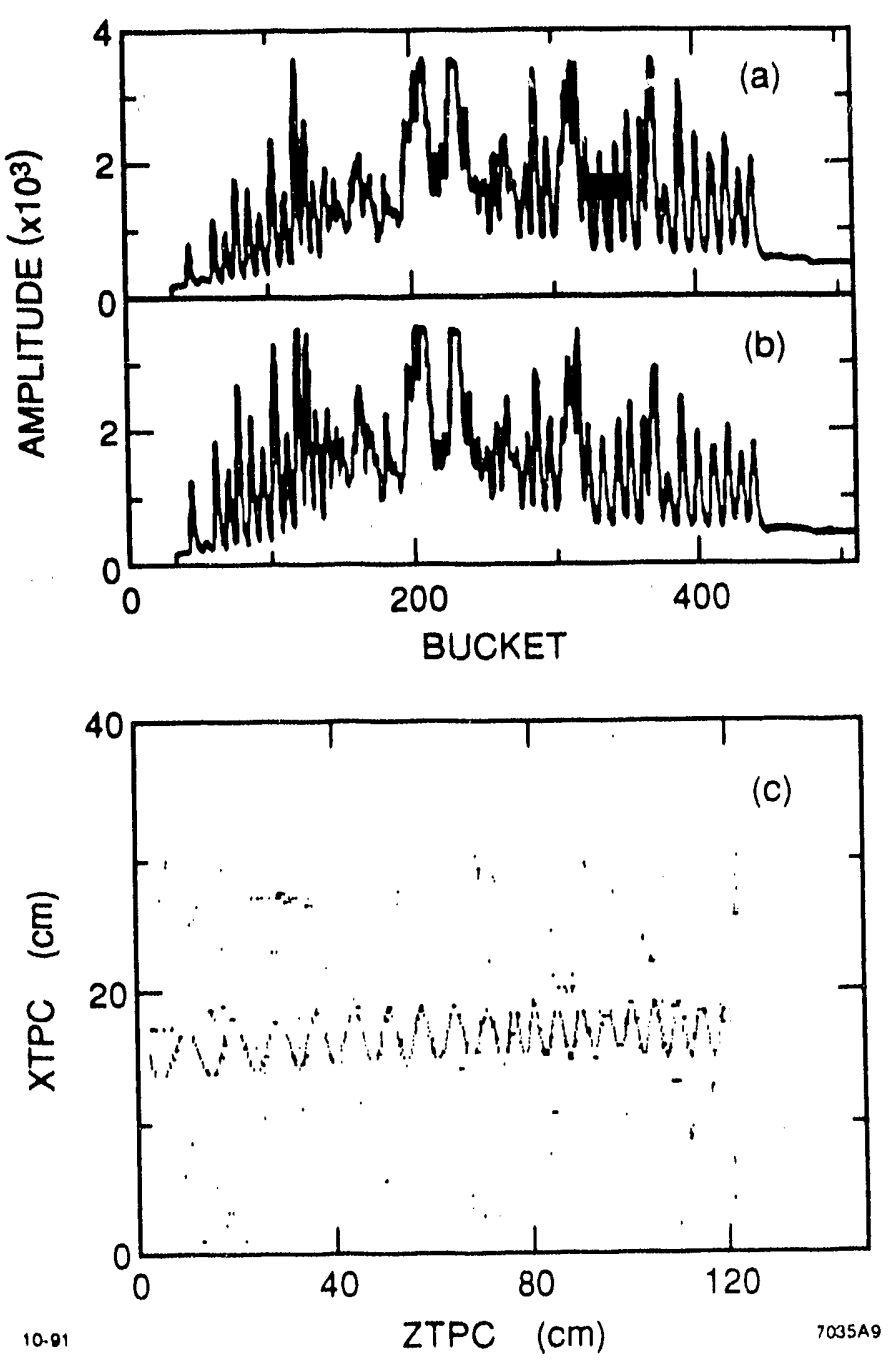

Fig. 7. (a) Inner and (b) Outer wire profiles for a low energy electron which is spiralling in the magnetic field. and (c) particle path after reconstruction demonstrating the robustness of the data acquisition algorithms.

[4] J. Hoeflich et al, "Design and Construction of the Front End Electronics Data Acquistion System for the SLD." IEEE Trans. Nucl. Sci. NS-38 No. 2, 348 (1991).

[5] K. Abe et al., "Initial Performance of the SLD Cerenkov Ring Imaging Detector System," submitted to this conference.

[6] E. Spencer et al., "Development of a Low Noise Preamplifier foi the Detection and Position Determination of Single Electrons in a Cerenkov Ring Imaging Detector by Charge Division," IEEE Trans. Nucl. Sci. NS-35, 23i (1988); SLAC-PUB-4404

(7) D. Freytag et al., IEEE Trans. Nucl. Sci. NS-33, 81 (1986).

[8] D. J. Sherden, "The Data Acquisition System for SLD." IEEE Trans. Nucl. Sci. NS-34, 142 (1987).

\section{DISCLAIMER}

This report was prepared as an account of work sponsored by an agency of the United States Government. Neither the United States Government nor any agency thereof, nor any of their employees, makes any warranty, express or implied, or assumes any legal liability or responsibility for the accuracy, completeness, or usefulness of any information, apparatus, product, or process disclosed, or represents that its use would not infringe privately nwned rights. Reference herein to any specific commercial product, process, or service by trade name, trademark, manufacturer, or otherwise does not necessarily constitute or imply its endorsement, recommendation, or favoring by the United States Government or any agency thereof. The views and opinions of authors expreseed herein to not necessarily state or reflect those of the United States Government or any agency thereof. 

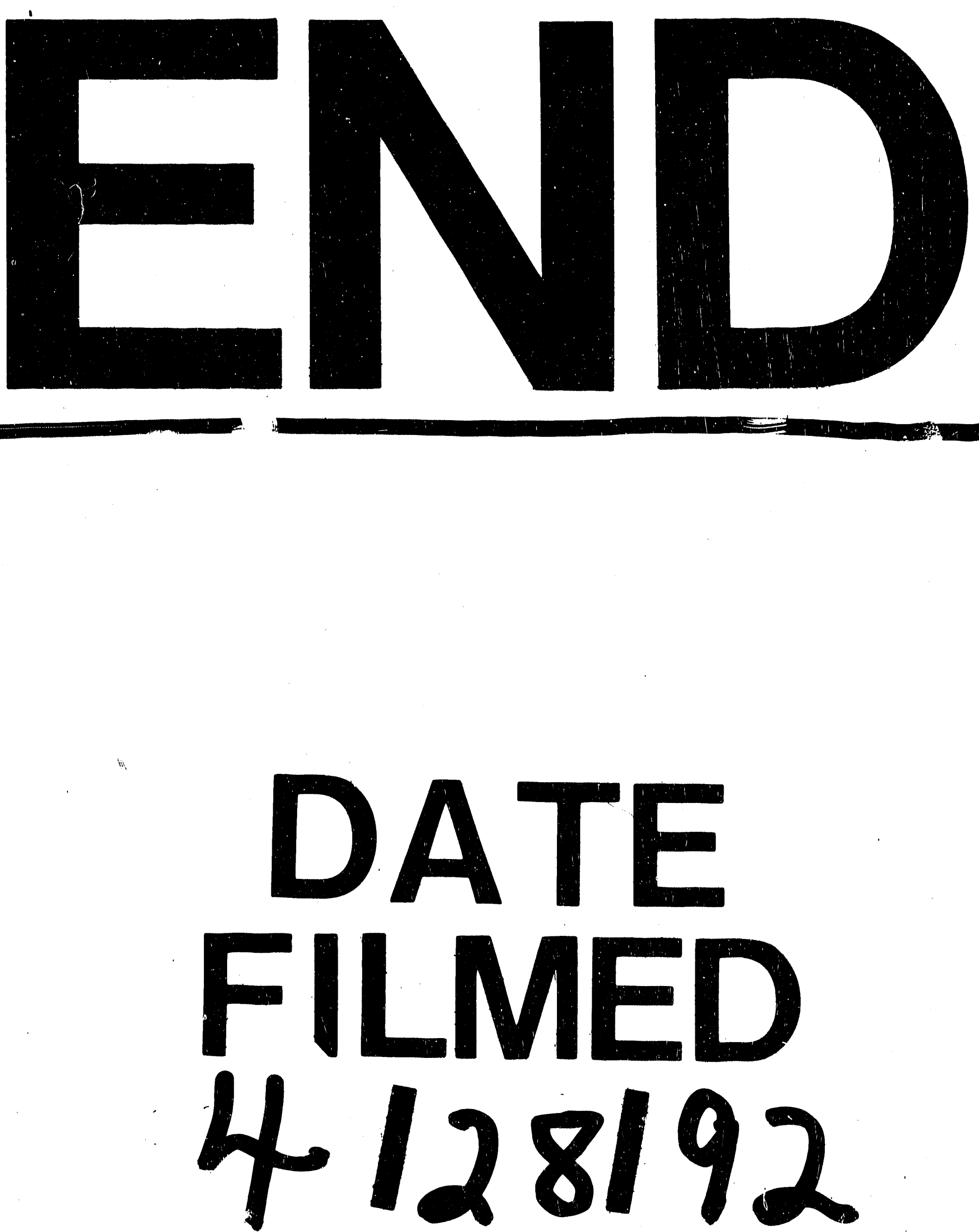
